\title{
Pontos positivos e negativos dos relatórios de sustentabilidade no modelo global reporting initiative: revisão da literatura nacional e internacional
}

Igor Laguna Vieira

Universidade do Estado do Rio de Janeiro (UERJ), Rio de Janeiro, RJ, Brasil

Elmo Rodrigues da Silva elmorodrigues@yahoo.com.br Universidade do Estado do Rio de Janeiro (UERJ), Rio de Janeiro, RJ, Brasil

\section{Luiz Carlos De Martini Junior} demartini@demartiniambiental.com.br Universidade do Estado do Rio de Janeiro (UERJ), Rio de Janeiro, RJ, Brasil

Ubirajara Aluizio de Oliveira Mattos

ubirajaraaluizio@yahoo.com.br Universidade do Estado do Rio de Janeiro (UERJ), Rio de Janeiro, RJ, Brasil

\author{
RESUMO
}

Considerando a crescente adesão de empresas ao redor do mundo pelo modelo de reporte da Global Reporting Initiative (GRI) para a produção de relatórios de sustentabilidade, o objetivo deste artigo é identificar pontos positivos e negativos desse modelo. Para tanto, procedeu-se à uma revisão da literatura, buscando-se artigos nacionais e internacionais em bases de dados constantes no Portal de Periódicos da Coordenação de Aperfeiçoamento de Pessoal de Nível Superior. Foram selecionados 55 artigos que traziam pontos positivos e/ou negativos dos relatórios de sustentabilidade no modelo de reporte da GRI. Observou-se que o periódico "Journal of Cleaner Production" se sobressaiu em relação ao número de publicações sobre o assunto, com 25 artigos do total. Os pontos positivos destacados na pesquisa foram agrupados nos seguintes tópicos: "Responsabilidade, transparência e legitimidade organizacional"; "Padronização e comparabilidade"; "Popularidade e credibilidade"; e "Abrangência". Já os pontos negativos destacados, em tópicos, foram: "Incompletude"; "Padronização pouco rígida e dificuldade em estabelecer comparações", "Greenwashing"; "Diferentes expectativas dos stakeholders"; "Alto custo de desenvolvimento do relatório"; e "Falta de integração entre indicadores". Buscou-se contribuir preenchendo uma lacuna na literatura sobre estudos desse tipo, que reunissem os pontos fortes e fracos do modelo GRI, observando-se ainda certos pontos de controvérsias entre autores. Como sugestão para pesquisas futuras, recomenda-se um aprofundamento em relação ao princípio da materialidade, considerado ponto crítico por alguns autores presentes nesta revisão e pouco abordado na literatura.
\end{abstract}

PALAVRAS-CHAVE: Desenvolvimento sustentável. Relatório de sustentabilidade. GRI. Responsabilidade social corporativa. 


\section{INTRODUÇÃO}

Desde o surgimento do conceito de "desenvolvimento sustentável", comumente definido como uma forma de desenvolvimento que atenda às necessidades do presente sem comprometer as gerações futuras (WCED, 1987), houve mudanças na relação das organizações com a questão da sustentabilidade. Outra importante abordagem, surgida posteriormente, é a do triple bottom line ou "tripé da sustentabilidade", cunhada por Elkington (1997), a qual considera o desempenho sustentável das empresas nas dimensões econômica, social e ambiental. Ao longo do tempo, gestores passaram a incorporar essas questões na estratégia das empresas (LACY et al., 2010), tendo essa mudança de paradigma ocorrido muito em virtude de pressões competitivas, do aumento do rigor legal e de demandas da sociedade (CORNELIUS; WALLACE; TASSABEHJI, 2007; MELÉ; DEBELUUH; ARRUDA, 2006; OLIVEIRA et al., 2013).

Mais recentemente, por meio da chamada Agenda 2030, a Organização das Nações Unidas (ONU) renovou esse chamado de atenção para questões de sustentabilidade, envolvendo, é claro, as organizações empresariais (BORGERT et al., 2018). Todavia, chama-se a atenção para o fato de o conceito de "desenvolvimento sustentável" possuir controvérsias, especialmente devido à natureza subjetiva das necessidades humanas e à complexa tarefa de identificar as condições necessárias para atendê-las (BJ ØRN et al., 2017).

Com relação ao meio empresarial, entende-se que, para avaliar o desempenho de uma organização em relação à sustentabilidade, tal desempenho deve ser mensurável (OZDEMIR et al., 2011). Como as partes interessadas (stakeholders) cada vez mais exigem divulgações por parte das organizações, vários modelos e métodos vêm sendo desenvolvidos e aprimorados. A esse exemplo, podem-se citar os relatórios de sustentabilidade corporativa.

A divulgação de informações relacionadas à sustentabilidade por parte das empresas vem crescendo e boa parte delas já possuem áreas dedicadas ao assunto em seus sites, incluindo a divulgação de relatórios (FERREIRA-QUILICE; CALDANA, 2015). Dentre as principais diretrizes para relatórios de sustentabilidade, destaca-se a da Global Reporting Initiative (GRI).

Com a crescente adesão de empresas ao modelo de reporte da GRI, indagase: como tal modelo de relatório vem sendo avaliado na literatura científica nacional e internacional, em termos de seus pontos positivos e negativos? Este artigo de revisão busca responder a esse questionamento identificando quais são esses pontos, por meio de revisão da literatura. A escolha pelas diretrizes da GRI ocorreu devido a essas possuírem amplo reconhecimento e aceitação internacional (PIMENTA; MORAIS, 2014; REBELATO; ALMEIDA; RODRIGUES, 2015).

Acredita-se que este trabalho possa contribuir para os estudos sobre o modelo de relatório da GRI, identificando os aspectos fortes e fracos, oferecendo oportunidades de melhoria a tal modelo. Embora seja numerosa a produção de artigos que abordam o GRI, busca-se suprir uma lacuna de estudos críticos que reúnam os pontos fortes e fracos desse modelo de reporte. Portanto, há uma oportunidade de avaliar a semântica de artigos científicos que tratam de relatórios de sustentabilidade segundo os padrões definidos pela GRI. 
Este artigo possui, além desta introdução, mais cinco outras seções principais. A segunda traz o Referencial Teórico da pesquisa. A terceira apresenta os procedimentos metodológicos utilizados para alcançar os objetivos delineados. A quarta apresenta os principais resultados, e na quinta, eles são discutidos. Por fim, são trazidas as considerações finais do artigo.

\section{REFERENCIAL TEÓRICO}

\section{RELATÓRIOS DE SUSTENTABILIDADE}

Os relatórios de sustentabilidade devem fornecer às partes interessadas informações que Ihes permitam avaliar o desempenho social e ambiental da organização (BOUTEN et al., 2011), viabilizando verificar se as ações de uma empresa estão alinhadas com seus valores e expectativas e, consequentemente, aprovar ou desaprovar as práticas organizacionais (SEARCY; BUSLOVICH, 2013). A transparência dessas informações melhora a credibilidade da empresa, favorecendo seus processos de criação de valor a longo prazo (SCHMELTZ, 2014). No entanto, são diversos fatores relacionados às características organizacionais que afetam os impactos socioambientais causados pela empresa e as expectativas das partes interessadas em relação à sustentabilidade, tais como: modelo de negócios, porte da empresa e contexto sociocultural em que se insere (PORTER; KRAMER, 2006). Por isso, nem todos os aspectos da sustentabilidade têm a mesma relevância para todas as empresas (MURILLO; LOZANO, 2006; ROBERTS; LAWSON; NICHOLLS, 2006).

Há também críticas com relação aos relatórios de sustentabilidade. Apontase que eles podem ser utilizados pelas empresas de forma eticamente reprovável para se mostrarem ambientalmente conscientes e proativas e, assim, legitimar seus negócios (MILNE; GRAY, 2012; ROBINSON, 2004). Brooks e Oikonomou (2018) alertam que empresas podem usar tais relatórios para praticar o chamado "greenwashing" (ou lavagem verde, em português), evidenciando aspectos em que possuem um bom desempenho, enquanto suprimem indicadores negativos, com o objetivo de transparecer uma imagem ecologicamente responsável, mas nem sempre verdadeira. Dessa forma, é fundamental que os relatórios de sustentabilidade sejam produzidos considerando, além dos resultados positivos do desempenho socioambiental da empresa, também os resultados negativos e possíveis formas de resolvê-los (DE MARTINI JUNIOR; SILVA; MATTOS, 2014).

\section{GLOBAL REPORTING INITIATIVE (GRI)}

Criada em 1997 em Amsterdã (Holanda), a GRI é uma organização sem fins lucrativos, fruto de um esforço conjunto da Coalition for Environmentally Responsible Economies (CERES) e do United Nations Environmental Program (UNEP), que visa auxiliar governos e organizações a compreender os impactos dos negócios no desenvolvimento sustentável (CAMPOS et al., 2013). A primeira versão das diretrizes da GRI para relato de sustentabilidade foi publicada em 2000. Desde então, regularmente, há revisão dessas diretrizes, chegando à quarta versão em 2013, conhecida como GRI G4 (BORGES et al., 2017). 
Em outubro de 2016, a GRI lançou a série GRI Standards. Desenvolvida pelo Global Sustainability Standards Board (GSSB), ela permite que organizações divulguem de forma pública seus impactos econômicos, ambientais e sociais, por meio de indicadores. Tal série inclui os principais conceitos das diretrizes GRI G4 aprimorados para uma estrutura mais flexível, além de requisitos mais claros e linguagem mais simples (GRI, 2019a).

Ainda em 2016, por recomendações da GRI, as organizações foram incentivadas a adotar o novo padrão (Standards) em seus relatórios de sustentabilidade. Após julho de 2018, obrigatoriamente, tanto os relatórios como outros materiais que fizerem referência à GRI deverão adotar esse novo modelo. Portanto, desde essa data, as diretrizes GRI G4 não podem mais ser utilizadas (GRI, 2018).

Segundo o Banco de Dados da GRI, desde a primeira versão de suas diretrizes, 13.943 organizações ao redor do mundo divulgaram seus relatórios seguindo o formato sugerido pela organização. No Brasil, esse número é de 485 empresas (GRI, 2019b).

\section{MATERIALIDADE}

Para a produção dos relatórios de sustentabilidade, as empresas precisam focar nas questões que são verdadeiramente críticas para o alcance das metas organizacionais e o gerenciamento dos impactos socioambientais. Isso significa dizer que elas devem adotar a chamada "materialidade" ao definir o conteúdo de seus relatórios (BEBBINGTON, 2001). Segundo a GRI (2015), "materialidade" é o limiar em que algum aspecto se torna realmente relevante para ser relatado. De acordo com esse princípio, os relatórios devem cobrir aspectos que representem os impactos econômicos, ambientais e sociais mais significativos da organização; ou aqueles que substancialmente influenciem as avaliações e decisões das partes interessadas. A partir da edição da GRI G4, as diretrizes da GRI para a produção de relatórios de sustentabilidade passaram a enfatizar a importância de focar nos aspectos "materiais" (MARIMON et al., 2012).

Entretanto, a avaliação da materialidade é um processo subjetivo, no qual os tomadores de decisão são solicitados a identificar, selecionar e priorizar questões de sustentabilidade para constar nos relatórios das empresas (HSU; LEE; CHAO, 2013; ZHOU; LI; HUANG, 2011). Segundo Calabrese et al. (2016), a literatura publicada contém poucos estudos que investigam a avaliação da materialidade para relatórios de sustentabilidade.

\section{METODOLOGIA}

Este artigo de revisão pode ser classificado como descritivo quanto aos objetivos, e bibliográfico quanto às fontes e aos procedimentos de coletas de dados (GIL, 2007).

O método aplicado nesta pesquisa será exposto nesta seção. A seguir são apresentadas as bases acadêmicas utilizadas, o período e os descritores de busca, os critérios de seleção dos artigos e a forma de obtenção de resultados. 
As bases de dados foram selecionadas incluindo-se algumas das principais coleções de artigos acadêmicos constantes no "Portal de Periódicos da Coordenação de Aperfeiçoamento de Pessoal de Nível Superior - CAPES", como a "Science Direct Journals (Elsevier)", a "Springer (CrossRef)", a "SCIELO (CrossRef)", a "SCIELO Brazil" e a "SCIELO".

Tal como acontece com todos os tipos de pesquisa, esta revisão da literatura tem certas limitações. Em primeiro lugar, foram incluídos nesta pesquisa apenas os artigos revisados por pares, publicados em periódicos acadêmicos indexados nas bases citadas acima, em língua inglesa ou portuguesa (ou seja, sem relatórios ou documentos da literatura "cinzenta", nem capítulos de livros, artigos de eventos, teses ou dissertações). $O$ ponto de partida para a revisão foi o ano 2014, logo após o surgimento das diretrizes G4 da GRI. O artigo mais recente incluído na pesquisa é de fevereiro de 2019.

Para a seleção dos artigos utilizou-se os descritores da seguinte forma: 1) "Global Reporting Initiative" AND "G4"; 2) "GRI Standards" NOT "G4". Por meio desses termos, buscou-se encontrar artigos que tratavam sobre relatórios GRI, mais especificamente das versões mais recentes (G4 ou Standards). A busca descrita na forma "2" se deu para evitar superposição de artigos já encontrados em " 1 ". Ao todo, encontrou-se 161 artigos.

Os artigos foram lidos na íntegra, excluindo-se aqueles que não abordavam qualquer ponto positivo ou negativo do modelo de relatório $\mathrm{GRI}$, ou seja, não atendiam ao objetivo desta pesquisa. Com isso foram selecionados 55 artigos.

\section{OBTENÇÃO DOS RESULTADOS}

Os artigos selecionados foram explorados e os pontos positivos e negativos citados para o modelo de relatório GRI foram destacados. Optou-se por dividir os pontos positivos em quatro categorias, por meio de método indutivo: "Responsabilidade, transparência e legitimidade organizacional"; "Padronização e comparabilidade"; "Popularidade e credibilidade"; e "Abrangência". Já os pontos negativos foram divididos nas seguintes categorias, também por indução: "Incompletude"; "Padronização pouco rígida e dificuldade em estabelecer comparações", "Greenwashing"; "Diferentes expectativas dos stakeholders"; "Alto custo de desenvolvimento do relatório"; e "Falta de integração entre indicadores". Estes pontos foram destacados uma vez que se repetiam nos artigos e serão explicitados e discutidos nas próximas seções.

\section{RESULTADOS}

\section{NÚMERO DE PUBLICAÇÕES POR ANO E POR PERIÓDICO}

Um total de 55 artigos foram incluídos nesta revisão. Todos esses artigos traziam pontos positivos e/ou negativos dos relatórios de sustentabilidade no modelo de reporte da GRI. 
O Quadro 1 apresenta o número total de publicações por ano, divididas por periódico. Como esta revisão incluiu apenas os artigos das bases de dados selecionadas nos primeiros dois meses de 2019 (ou seja, apenas um artigo), espera-se que mais artigos ainda sejam publicados sobre o tema no ano.

Quadro 1 - Número de artigos por periódico e ano

\begin{tabular}{|c|c|c|c|c|c|c|c|}
\hline $\begin{array}{c}\text { Ano } \rightarrow \\
\text { Periódico } \downarrow\end{array}$ & 2014 & 2015 & 2016 & 2017 & 2018 & 2019 & $\begin{array}{c}\text { TOTAL POR } \\
\text { REVISTA }\end{array}$ \\
\hline Applied Ergonomics & - & - & 1 & - & - & - & 1 \\
\hline Brazilian Business Review & - & - & - & - & 1 & - & 1 \\
\hline $\begin{array}{c}\text { Computers and Chemical } \\
\text { Engineering }\end{array}$ & - & 1 & - & - & - & - & 1 \\
\hline Convergencia & - & - & - & 1 & - & - & 1 \\
\hline $\begin{array}{c}\text { Critical Perspectives on } \\
\text { Accounting }\end{array}$ & - & 2 & - & - & - & - & 2 \\
\hline $\begin{array}{c}\text { Environmental Impact } \\
\text { Assessment Review }\end{array}$ & - & - & - & - & 1 & - & 1 \\
\hline Estudios Gerenciales & - & - & - & 1 & - & - & 1 \\
\hline Forest Policy and Economics & - & - & - & 1 & - & - & 1 \\
\hline Gestão e Produção & - & 1 & - & 1 & - & - & 2 \\
\hline $\begin{array}{c}\text { Journal of Information } \\
\text { Systems and Technology } \\
\text { Management }\end{array}$ & - & - & - & 1 & - & - & 1 \\
\hline Journal of Business Ethics & - & - & - & 1 & 1 & - & 2 \\
\hline $\begin{array}{c}\text { Journal of Cleaner } \\
\text { Production }\end{array}$ & 5 & 1 & 4 & 7 & 8 & - & 25 \\
\hline $\begin{array}{c}\text { Journal of Environmental } \\
\text { Manegement }\end{array}$ & - & 1 & - & - & - & - & 1 \\
\hline Minerals Engineering & - & - & - & 1 & - & - & 1 \\
\hline Produção & 1 & - & - & - & - & - & 1 \\
\hline Quality e Quantity & - & - & - & - & 1 & - & 1 \\
\hline $\begin{array}{c}\text { RAUSP Management Journal } \\
\text { (antiga Revista de } \\
\text { Administração) }\end{array}$ & - & 1 & - & 1 & 1 & - & 3 \\
\hline Renewable Energy & - & - & - & - & - & 1 & 1 \\
\hline Resources Policy & - & 1 & - & - & 1 & - & 2 \\
\hline $\begin{array}{l}\text { Revista Contabilidade e } \\
\text { Finanças }\end{array}$ & 1 & - & - & - & - & - & 1 \\
\hline Revista de Contabilidad & - & 1 & 1 & - & - & - & 2 \\
\hline $\begin{array}{c}\text { Revista de Gestão dos Países } \\
\text { de Língua Portuguesa }\end{array}$ & 1 & - & - & - & - & - & 1 \\
\hline Safety Science & 1 & - & - & - & - & - & 1 \\
\hline $\begin{array}{c}\text { The British Accounting } \\
\text { Review }\end{array}$ & 1 & - & - & - & - & - & 1 \\
\hline TOTAL POR ANO & 10 & 9 & 6 & 15 & 15 & 1 & - \\
\hline TOTAL GERAL & - & - & - & - & - & - & 55 \\
\hline
\end{tabular}

Fonte: Autoria própria (2019)

O "Journal of Cleaner Production" publicou a maior parte dos artigos incluídos nesta revisão ( 25 de um total de 55 artigos), com maior destaque para os anos de 2017 e 2018 (sete e nove artigos, respectivamente). Interessante observar que esses anos também se destacaram no total, com quinze artigos em cada um deles. 
A "RAUSP Management Journal" (antiga "Revista de Administração") publicou três artigos. Dos que publicaram dois artigos cada estão os periódicos: "Critical Perspectives on Accounting"; "Gestão e Produção"; "Journal of Business Ethics"; "Resources Policy"; e "Revista de Contabilidad". As demais revistas incluídas na revisão publicaram um artigo cada.

\section{PONTOS POSITIVOS E NEGATIVOS DOS RELATÓRIOS GR}

Por meio da leitura dos artigos, os pontos positivos e/ou negativos apontados foram categorizados conforme exposto na seção "OBTENÇÃO DOS RESULTADOS". No Quadro 2 é apontado um resumo dos achados para os pontos positivos, enquanto no Quadro 3 faz-se o mesmo para os negativos. Para cada categoria é trazida uma breve descrição de seu significado e quais referências abordaram esses pontos em suas pesquisas. É apresentado ainda o quantitativo dos trabalhos que apresentam tais pontos, com seu percentual representativo do total de artigos incluídos nesta revisão.

Quadro 2 - Pontos positivos

\begin{tabular}{|c|c|c|c|}
\hline Categoria & Descrição & Autores & Quantitativo \\
\hline $\begin{array}{c}\text { Responsabilidade, } \\
\text { transparência e } \\
\text { legitimidade } \\
\text { organizacional }\end{array}$ & $\begin{array}{c}\text { Os relatórios GRI } \\
\text { auxiliam as empresas na } \\
\text { busca de uma gestão que } \\
\text { considere o } \\
\text { desenvolvimento } \\
\text { sustentável, aumentando } \\
\text { a credibilidade delas em } \\
\text { relação ao tema. Eles } \\
\text { ainda promovem a } \\
\text { transparência e a } \\
\text { responsabilidade } \\
\text { organizacional. }\end{array}$ & $\begin{array}{l}\text { Adams (2015); Aras, } \\
\text { Tezcan e Furtuna } \\
\text { (2018); Borgert et al. } \\
\text { (2018); Burgwal e } \\
\text { Vieira (2014); } \\
\text { Calabrese et al. (2016); } \\
\text { Dissanayake, Tilt e } \\
\text { Xydias-Lobo (2016); } \\
\text { Fedorova e Pongrácz } \\
\text { (2019); Ferreira-Quilice } \\
\text { e Caldana (2015); } \\
\text { Fonseca, McAllister e } \\
\text { Fitzpatrick (2014); Fritz, } \\
\text { Schöggl e Baumgartner } \\
\text { (2017); } \\
\text { Fuisz-Kehrbach (2015); } \\
\text { González et al. (2018); } \\
\text { Liubachyna, Secco e } \\
\text { Pettenella (2017); Lock } \\
\text { e Seele (2016); Mancini } \\
\text { e Sala (2018); } \\
\text { Michelon, Pilonato e } \\
\text { Ricceri (2015); Siew } \\
\text { (2015); Talbot e Boiral } \\
\text { (2018); Silvestre e } \\
\text { Amaro (2014); Smith, } \\
\text { Ruiz-Mercado e } \\
\text { Gonzalez (2015); } \\
\text { Soares et al. (2018); } \\
\text { Tsalis, Avramidou e } \\
\text { Nikolaou (2017); Vogt } \\
\text { et al. (2017); Weber e } \\
\text { Saunders-Hogberg } \\
\text { (2018) }\end{array}$ & $23(41,82 \%)$ \\
\hline
\end{tabular}




\begin{tabular}{|c|c|c|c|}
\hline $\begin{array}{l}\text { Padronização e } \\
\text { comparabilidade }\end{array}$ & $\begin{array}{c}\text { As diretrizes GRI } \\
\text { possibilitam a } \\
\text { padronização no } \\
\text { conteúdo dos relatórios, } \\
\text { permitindo comparação } \\
\text { com as práticas e } \\
\text { desempenho de outras } \\
\text { organizações. }\end{array}$ & $\begin{array}{l}\text { Calabrese et al. (2016); } \\
\text { Dissanayake, Tilt e } \\
\text { Xydias-Lobo (2016); } \\
\text { González et al. (2018); } \\
\text { Lock e Seele (2016); } \\
\text { Scholl, Hourneaux } \\
\text { Junior e Galleli (2015); } \\
\text { Soares et al. (2018); } \\
\text { Talbot e Boiral (2018); } \\
\text { Tsalis, Avramidou e } \\
\text { Nikolaou (2017); } \\
\text { Wagner e Seele (2017); } \\
\text { Weber e Saunders- } \\
\text { Hogberg (2018) }\end{array}$ & $10(18,18 \%)$ \\
\hline $\begin{array}{c}\text { Popularidade e } \\
\text { credibilidade }\end{array}$ & $\begin{array}{c}\text { A Global Reporting } \\
\text { Initiative produz as } \\
\text { principais diretrizes } \\
\text { internacionalmente } \\
\text { aceitas para relatórios de } \\
\text { sustentabilidade, sendo a } \\
\text { mais utilizada. }\end{array}$ & $\begin{array}{c}\text { Alcaraz-Quiles, } \\
\text { Navarro-Galera e Ortiz- } \\
\text { Rodrígues (2017); } \\
\text { Arena, Azzone e } \\
\text { Mapelli (2018); Blass et } \\
\text { al. (2017); Bonsón e } \\
\text { Bednárová (2015); } \\
\text { Brusca, Labrador e } \\
\text { Larran (2018); Burritt e } \\
\text { Schaltegger (2014); } \\
\text { Danoucaras, Woodley e } \\
\text { Moran (2014); Dilling e } \\
\text { Harris (2018); } \\
\text { Dissanayake, Tilt e } \\
\text { Xydias-Lobo (2016); } \\
\text { Dragomir (2018); Etzion } \\
\text { et al. (2017); } \\
\text { Fernandez-Feijoo, } \\
\text { Romero e Ruiz (2014); } \\
\text { Ferreira-Quilice e } \\
\text { Caldana (2015); Fifka e } \\
\text { Pobizhan (2014); } \\
\text { Fonseca, McAllister e } \\
\text { Fitzpatrick (2014); } \\
\text { Fuente, Garcí-Sánchez } \\
\text { e Lozano (2017); } \\
\text { González et al. (2018); } \\
\text { Jackson e Belkhir } \\
\text { (2018); Koskela (2014); } \\
\text { Lima et al. (2016); } \\
\text { Liubachyna, Secco e } \\
\text { Pettenella (2017); Lock } \\
\text { e Seele (2016); } \\
\text { Machado et al. (2017); } \\
\text { Mascena, Fischmann e } \\
\text { Boaventura (2018); } \\
\text { Michelon, Pilonato e } \\
\text { e Carvalho (2017); } \\
\text { Nascimento, Araujo e } \\
\text { Alves (2017); Oliveira et }\end{array}$ & $40(72,73 \%)$ \\
\hline
\end{tabular}




\begin{tabular}{|c|c|c|c|}
\hline & & $\begin{array}{c}\text { al. (2014); Rahdari e } \\
\text { Rostamy (2015); Ruiz- } \\
\text { Lozano e Tirado- } \\
\text { Valencia (2016); } \\
\text { Samudhram et al. } \\
\text { (2016); Scholl, } \\
\text { Hourneaux Junior e } \\
\text { Galleli (2015); Silvestre } \\
\text { e Amaro (2014); Souza } \\
\text { e Alves (2018); Spuerk, } \\
\text { Drobe e Lottermoser } \\
\text { (2017); Talbot e Boiral } \\
\text { (2018); Tsalis, } \\
\text { Avramidou e Nikolaou } \\
\text { (2017); Veleva, Bodkin } \\
\text { e Todorova (2017); } \\
\text { Vogt et al. (2017); } \\
\text { Wagner e Seele (2017) }\end{array}$ & \\
\hline Abrangência & $\begin{array}{c}\text { Os padrões da GRI } \\
\text { fornecem diversos } \\
\text { indicadores para que as } \\
\text { empresas possam relatar } \\
\text { seu desempenho } \\
\text { econômico, ambientale } \\
\text { social. }\end{array}$ & $\begin{array}{c}\text { Alcaraz-Quiles, } \\
\text { Navarro-Galera e Ortiz- } \\
\text { Rodrígues (2017); } \\
\text { Borgert et al. (2018); } \\
\text { Fuisz-Kehrbach e Sonja- } \\
\text { Katrin (2015); } \\
\text { Samudhram et al. } \\
\text { (2016); Talbot e Boiral } \\
\text { (2018) }\end{array}$ & $05(9,09 \%)$ \\
\hline
\end{tabular}

Fonte: Autoria própria (2019)

Quadro 3 - Pontos negativos

\begin{tabular}{|c|c|c|c|}
\hline Categoria & Descrição & Autores & Quantitativo \\
\hline Incompletude & $\begin{array}{c}\text { Ausência de certos } \\
\text { indicadores. }\end{array}$ & $\begin{array}{c}\text { Adams (2015); Mancini } \\
\text { e Sala (2018); Spuerk, } \\
\text { Drobe e Lottermoser } \\
\text { (2017); Veleva, Bodkin } \\
\text { e Todorova (2017) }\end{array}$ & 04 (7,27\%) \\
\hline $\begin{array}{l}\text { Padronização } \\
\text { pouco rígida e } \\
\text { dificuldade em } \\
\text { estabelecer } \\
\text { comparações }\end{array}$ & $\begin{array}{l}\text { Ausência de rigidez nas } \\
\text { diretrizes para } \\
\text { desenvolvimento dos } \\
\text { relatórios, o que não } \\
\text { garante a } \\
\text { comparabilidade entre } \\
\text { empresas. }\end{array}$ & $\begin{array}{l}\text { Danoucaras, Woodley e } \\
\text { Moran (2014); Etzion et } \\
\text { al. (2017); Ferreira- } \\
\text { Quilice e Caldana } \\
\text { (2015); Jackson e } \\
\text { Belkhir (2018); } \\
\text { Liubachyna, Secco e } \\
\text { Pettenella (2017); Siew } \\
\text { (2015); Spuerk, Drobe e } \\
\text { Lottermoser (2017); } \\
\text { Talbot e Boiral (2018) }\end{array}$ & $08(14,55 \%)$ \\
\hline Greenwashing & $\begin{array}{c}\text { As diretrizes de relato } \\
\text { podem ser usadas de } \\
\text { maneira tendenciosa, } \\
\text { como instrumento de } \\
\text { marketing. }\end{array}$ & $\begin{array}{c}\text { Bjørn et al. (2017); } \\
\text { Ferreira-Quilice e } \\
\text { Caldana (2015); } \\
\text { Jackson e Belkhir } \\
\text { (2018); Liubachyna, } \\
\text { Secco e Pettenella } \\
\text { (2017); Samudhram et } \\
\text { al. (2016); Siew (2015); }\end{array}$ & $07(12,73 \%)$ \\
\hline
\end{tabular}




\begin{tabular}{|c|c|c|c|}
\hline & & Talbot e Boiral (2018) & \\
\hline $\begin{array}{c}\text { Diferentes } \\
\text { expectativas dos } \\
\text { stakeholders }\end{array}$ & $\begin{array}{c}\text { Há diferentes partes } \\
\text { interessadas envolvidas, } \\
\text { cada uma com diferentes } \\
\text { expectativas, e há } \\
\text { dificuldade em atender a } \\
\text { todas. }\end{array}$ & $\begin{array}{c}\text { Adams (2015); } \\
\text { Calabrese et al. (2016); } \\
\text { Fonseca, McAllister e } \\
\text { Fitzpatrick (2014); } \\
\text { Leong et al. (2014); } \\
\text { Liubachyna, Secco e } \\
\text { Pettenella (2017); Siew } \\
\text { (2015) }\end{array}$ & $06(10,91 \%)$ \\
\hline $\begin{array}{c}\text { Alto custo de } \\
\text { desenvolvimento } \\
\text { do relatório }\end{array}$ & $\begin{array}{c}\text { Muitas organizações } \\
\text { deixam de divulgar o } \\
\text { relatório de } \\
\text { sustentabilidade ou } \\
\text { divulgam de forma } \\
\text { incompleta, sendo um } \\
\text { dos motivos o alto custo } \\
\text { para a obtenção de } \\
\text { informações. }\end{array}$ & $\begin{array}{c}\text { Calabrese et al. (2016); } \\
\text { Ferreira-Quilice e } \\
\text { Caldana (2015) }\end{array}$ & $02(3,64 \%)$ \\
\hline $\begin{array}{c}\text { Falta de integração } \\
\text { entre indicadores }\end{array}$ & $\begin{array}{c}\text { A estrutura da GRI } \\
\text { desconsidera os efeitos } \\
\text { interativos entre os } \\
\text { indicadores, correndo o } \\
\text { risco de perder de vista o } \\
\text { quadro geral da } \\
\text { sustentabilidade. }\end{array}$ & $\begin{array}{l}\text { Fonseca, McAllister e } \\
\text { Fitzpatrick (2014); } \\
\text { Samudhram et al. } \\
\text { (2016); Siew (2015) }\end{array}$ & $03(5,45 \%)$ \\
\hline
\end{tabular}

Fonte: Autoria própria (2019)

A seguir cada um dos pontos positivos e negativos citados acima serão melhor explicados e discutidos.

\section{Pontos Positivos}

Nesta seção, cada um dos pontos positivos do modelo de reporte da GRI apontados acima serão explicitados, salientando-se a visão de alguns dos autores que fizeram parte desta revisão.

\section{Responsabilidade, transparência e legitimidade organizacional}

Segundo Calabrese et al. (2016), a prática de medir e divulgar o desempenho de sustentabilidade apoia empresas na mudança para uma operação mais sustentável. Além disso, favorece uma comunicação mais transparente, o que tende a melhorar sua credibilidade. Por meio de relatórios de sustentabilidade, as partes interessadas podem estabelecer se as ações de uma empresa estão alinhadas com seus valores e expectativas e, consequentemente, aprovar ou desaprovar suas atividades. Essa abordagem permitiu criar as diretrizes de relato de sustentabilidade da GRI (LIUBACHYNA; SECCO; PETTENELLA, 2017).

A GRI baseia-se em pressupostos amplamente aceitos: o empoderamento e mobilização de atores sociais motivados pela busca de informação, demandando prestação de contas por parte das organizações; o interesse das organizações em atender demandas da sociedade, criando uma imagem de responsabilidade e transparência; e a integração entre vários stakeholders como forma de 
governança colaborativa para sustentabilidade (FERREIRA-QUILICE; CALDANA, 2015).

Segundo Calabrese et al. (2016), a abordagem de materialidade nos relatórios de sustentabilidade melhora os relacionamentos entre as partes interessadas e as empresas, contribuindo para processos de criação de valor compartilhado, conectando a sociedade ao progresso econômico. Ainda de acordo com o autor, o uso das diretrizes da GRI aumenta a responsabilidade das empresas, uma vez que elas são demandadas a relatar os efeitos positivos e negativos de suas ações.

Outro benefício da estrutura de relatório da GRI é o fato de que empresas que adotam tais diretrizes tendem a reduzir, de forma significativa, tempo e esforços gastos respondendo às demandas sobre informações sociais e ambientais, uma vez que os relatórios são públicos (SIEW, 2015). As diretrizes da GRI podem ainda servir como insumo para a elaboração de um sistema adequado de mensuração de desempenho de sustentabilidade para a tomada de decisão interna (ARAS; TEZCAN; FURTUNA, 2018).

Por meio das diretrizes da GRI as empresas podem passar de uma visão social ou ambiental da sustentabilidade para uma visão mais integrada (DISSANAYAKE; TILT; XYDIAS-LOBO, 2016). As diretrizes da GRI têm o potencial de melhorar significativamente a utilidade e a qualidade das informações relatadas pelas empresas em relação a seus impactos e desempenho ambientais, sociais e econômicos (GONZÁLEZ et al. 2018).

\section{Padronização e comparabilidade}

Como resultado do surgimento do GRI, as práticas de divulgação ambiental tornaram-se cada vez mais padronizadas, favorecendo a ideia de que informações relevantes e transparentes estão disponíveis nos relatórios de sustentabilidade (TALBOT; BOIRAL, 2018). Segundo Calabrese et al. (2016), as diretrizes da GRI são apropriadas para empresas de todas as dimensões e setores, permitindo a comparação com as práticas e desempenho de outras organizações, uma vez que os relatórios são padronizados. Dissanayake, Tilt e Xydias-Lobo (2016) inclusive observa que a comparabilidade é um dos fatores que leva empresas a utilizarem as diretrizes da GRI, indicando ainda outros benefícios, como clareza e utilidade.

Além da possibilidade de se utilizar o benchmarking e realizar avaliação do desempenho de sustentabilidade, as diretrizes da GRI permitem às empresas a comparação do desempenho dentro de si própria ou em relação a outras organizações ao longo do tempo, uma vez que o formato é um padrão reconhecido mundialmente (SCHOLL; HOURNEAUX JUNIOR; GALLELI, 2015; SOARES et al., 2018).

\section{Popularidade e credibilidade}

Segundo Siew (2015), estudos vêm mostrando que empresas que desenvolvem seus relatórios com base na GRI vêm obtendo maior credibilidade em relação à qualidade de seus relatórios do que aquelas que não utilizam tal 
modelo. Além disso, os usuários da GRI possuem, em média, menor volatilidade no preço das ações e melhores margens de lucro operacional. Ainda segundo o autor, uma possível explicação para isso poderia ser a de que os analistas financeiros obtêm melhores previsões como resultado direto da transparência organizacional.

O sucesso da GRI é corroborado pelo fato de que dezenas de países e bolsas de valores implementaram diretrizes obrigatórias de divulgação de sustentabilidade, algumas das quais referenciam explicitamente as diretrizes da GRI (ETZION et al., 2017). Devido a esse sucesso, empresas utilizam os relatórios GRI como forma de estabelecer benchmarking e comparações (SCHOLL; HOURNEAUX JUNIOR; GALLELI, 2015). Para a indústria da mineração, por exemplo, Danoucaras, Woodley e Moran (2014) e Spuerk, Drobe e Lottermoser (2017) afirmam que a GRI é a iniciativa mais amplamente divulgada quando o assunto é relatório de sustentabilidade, tornando-se um padrão de facto para o setor.

\section{Abrangência}

As diretrizes de sustentabilidade GRI consideram questões nas três dimensões da sustentabilidade - econômica, ambiental e social - e identificam uma série de questões específicas de cada setor que devem ser relatadas. Isso se alinha com o uso de uma abordagem que identifique quais questões de sustentabilidade devem ser consideradas em sua avaliação, ou seja, o princípio da materialidade (BORGERT et al., 2018).

Os padrões GRI oferecem diversos indicadores para avaliar o desempenho de sustentabilidade de empresas, incluindo questões como direitos humanos, práticas trabalhistas e trabalho decente, sociedade e responsabilidade pelo produto (FUISZ-KEHRBACH; SONJA-KATRIN, 2015; SAMUDHRAM et al., 2016). Exemplificando a abrangência dos indicadores, Fuisz-Kehrbach e Sonja-Katrin (2015) consideram que as divulgações da GRI possuem boa cobertura em relação ao uso da terra e aspectos territoriais, com indicadores de disputas relacionadas à terra e reassentamentos.

\section{Pontos Negativos}

Analogamente ao que foi feito na seção "Pontos Positivos", nesta seção serão levantados os pontos negativos relativos às diretrizes GRI na visão de alguns autores presentes nesta revisão.

\section{Incompletude}

Alguns autores relatam a ausência de certos indicadores importantes nas diretrizes da GRI. Ferreira-Quilice e Caldana (2015) citam que as informações presentes nos relatórios de sustentabilidade GRI não são tão úteis aos problemas específicos do ativismo social, não dando uma visão adequada dos impactos nas comunidades locais. Mancini e Sala (2018) alegam que os impactos ambientais que afetam a saúde humana são apenas parcialmente incorporados nos indicadores ambientais. No caso da indústria da mineração, os impactos 
negativos na saúde e segurança das comunidades (incluindo, por exemplo, danos devidos a explosivos e problemas de saúde mental devido a ciclos de expansão/recessão) também não estão incorporados nas diretrizes GRI.

Ainda no setor da mineração, o esgotamento de depósitos minerais também não é coberto pela estrutura da GRI, nem é comumente tratado em relatórios de sustentabilidade (FONSECA; MCALLISTER; FITZPATRICK, 2014). Algumas empresas informam a quantidade de minério extraída no indicador sobre "materiais usados por peso" (massa). No entanto, Spuerk, Drobe e Lottermoser (2017) consideram inadequado avaliar a eficiência de recursos na mineração por meio de indicadores de entrada de material.

\section{Padronização pouco rígida e dificuldade em estabelecer comparações}

O estudo de Ferreira-Quilice e Caldana (2015) mostra que algumas organizações demonstram dificuldades na utilização do modelo proposto. Podese citar: protocolo pouco específico; indicadores de difícil interpretação; relatório genérico e distante de aspectos locais; e ausência de padronização. Para os autores, devido à falta de padronização dos relatórios, não se torna possível a comparação entre organizações, ainda que do mesmo setor, o que também é corroborado por Siew (2015).

Liubachyna, Secco e Pettenella (2017) ressaltam a necessidade de desenvolver metodologias mais claras para relatar o progresso da sustentabilidade da empresa, uma vez que, embora a GRI tenha fornecido diretrizes para relatórios de sustentabilidade, ela não estipulou modelos, não aplicou a conformidade nem se envolveu diretamente na auditoria, gerando uma heterogeneidade significativa nos relatórios que adotam o seu modelo (ETZION et al., 2017).

\section{Greenwashing}

Siew (2015) adverte que as diretrizes da GRI promovem uma abordagem "gerencialista" (ou empresarial) da sustentabilidade e isso pode levar as empresas a uma "armadilha avaliativa", com grande parte dos relatórios de sustentabilidade sendo utilizada como uma ferramenta para ocultar práticas reais. Liubachyna, Secco e Pettenella (2017) ressaltam a necessidade em verificar se os relatórios estão alinhados com o conceito de responsabilidade corporativa e representar os impactos reais das atividades da empresa.

Siew (2015) destaca como um dos principais problemas dos relatórios de sustentabilidade 0 fato de que algumas corporações manipulam deliberadamente a percepção das partes interessadas por meio do greenwashing.

\section{Diferentes expectativas dos stakeholders}

Os relatórios de sustentabilidade no modelo GRI oferecem oportunidade para que as partes interessadas possam avaliar o desempenho de uma organização, auxiliando na tomada de decisões baseadas na forma de como a 
empresa influencia o ambiente e a sociedade. No entanto, as diferentes partes interessadas possuem expectativas diversas, sendo um dos maiores desafios apresentar informações correspondentes a todas elas (Liubachyna, Secco e Pettenella, 2017).

Calabrese et al. (2016) observou a falta de métodos para resolver o problema da subjetividade inerente à análise de materialidade. Ferreira-Quilice e Caldana (2015) apontam como uma das principais dificuldades na utilização do modelo GRI justamente a de obter amostra relevante de stakeholders para elaborar uma matriz de materialidade.

Fonseca, McAllister e Fitzpatrick (2014) analisou relatórios de sustentabilidade de empresas de mineração, revelando que o princípio da materialidade tem sido abordado sem muitos detalhes de como a análise junto às partes interessadas foi realizada.

\section{Alto custo de desenvolvimento do relatório}

Ferreira-Quilice e Caldana (2015) afirmam que muitas organizações não disponibilizam todas as informações requeridas pelo modelo de reporte da GRI, sendo um dos motivos o alto custo para sua obtenção, por serem difíceis de medir e sem grande utilidade para a empresa. Outras dificuldades apontadas pelos autores foram a falta de tempo do pessoal interno para fornecimento das informações e a falta de confiabilidade nos dados levantados. Com isso, os autores comentam que o modelo proposto pela GRI atenderia mais as grandes empresas, que teriam mais recursos para desenvolver os relatórios.

\section{Falta de integração entre indicadores}

Siew (2015) analisou o modelo de relatório da GRI e concluiu que há um desequilíbrio de ênfase entre critérios econômicos, sociais e ambientais. Segundo o autor, o modelo ainda obscurece a aquisição de uma visão integrada da sustentabilidade do negócio e contribui para a percepção do conceito de desenvolvimento sustentável a partir de uma abordagem reducionista, esquecendo a interação entre os indicadores, o que também é apontado por Fonseca, McAllister e Fitzpatrick (2014). Para este último, como resultado dessa falta de interação, os relatórios de sustentabilidade têm perdido o "quadro geral" e correm o risco de informar mal os tomadores de decisão. Samudhram et al. (2016) também observam que as empresas vêm aplicando os padrões GRI de uma maneira não integrada.

\section{DISCUSSÃO}

O tema "GRI" mostra-se aplicável a diversos campos de pesquisa, haja vista a diversidade de revistas que publicam sobre o assunto. Apesar do destaque para a revista "Journal of Cleaner Production" (25 publicações de 55), que traz artigos sobre "produção limpa", há estudos publicados em revistas com diversos focos, a exemplo das seguintes áreas: ergonomia, indústria mineral, contabilidade, computação e engenharia química, ética corporativa e, é claro, meio ambiente. 
Dos pontos positivos, aquele mais citado nos artigos incluídos nesta revisão foi "Popularidade e credibilidade", sendo consenso o fato de que a GRI produz as diretrizes mais utilizadas no mundo para se produzir relatórios de sustentabilidade, corroborando Pimenta e Morais (2014) e Rebelato, Almeida e Rodrigues (2015). O segundo ponto mais citado foi "Responsabilidade, transparência e legitimidade organizacional", mostrando a importância de as organizações relatarem a sua relação com a questão da sustentabilidade, aumentando a sua credibilidade junto às partes interessadas, legitimando suas atividades, o que também é dito por Schmeltz (2014).

"Padronização pouco rígida e dificuldade em estabelecer comparações" foi o ponto negativo mais citado pelos autores incluídos nesta revisão, seguido de "Greenwashing". Há preocupação de alguns autores com relação ao fato de que os relatórios podem não traduzir a realidade do que ocorre nas corporações, sendo usados meramente como um instrumento de marketing. Até mesmo pela flexibilidade das diretrizes GRI, fica possibilitado "mascarar" certas práticas, uma vez que a questão da "materialidade" nem sempre é muito bem explicitada nos relatórios de sustentabilidade, permitindo que empresas divulguem apenas aquilo que as interessa, o que já era alertado por autores como Milne e Gray (2012), Robinson (2004), Brooks e Oikonomou (2018) e De Martini Junior, Silva e Mattos (2014).

A questão da materialidade ainda é citada no ponto negativo "Diferentes expectativas dos stakeholders", uma vez que pode acontecer de as partes interessadas terem interesses muito divergentes, às vezes conflituosos, e não é tarefa simples atender a todos, ressaltando a subjetividade destacada por Hsu, Lee e Chao (2013) e Zhou, Li e Huang (2011).

Enquanto alguns autores indicam como ponto positivo o fato de os relatórios GRI terem um certo padrão e, com isso, possibilitarem estabelecer comparações, outros autores expõem que a padronização é pouco rígida e isso impossibilitaria comparar relatórios. Liubachyna, Secco e Pettenella (2017) e Etzion et al. (2017) sugerem 0 desenvolvimento de metodologias menos amplas para o desenvolvimento de relatórios de sustentabilidade, estipulando modelos mais rígidos. De fato, dessa forma a comparabilidade seria favorecida.

Alguns autores apontam como vantagem do modelo GRI a amplitude de indicadores, abrangendo bem o tripé da sustentabilidade (ELKINGTON, 1997). No entanto, outros autores mostraram sentir falta de alguns indicadores, a exemplo de Ferreira-Quilice e Caldana (2015), que indicaram a ausência de indicadores para questões de ativismo social, e Fonseca, McAllister e Fitzpatrick (2014), que sentiram falta de indicadores para relatar o esgotamento de depósitos minerais. Ainda com relação aos indicadores, alguns autores, como Siew (2015), Fonseca, McAllister e Fitzpatrick (2014) e Samudhram et al. (2016), observam que as empresas vêm aplicando os padrões GRI de uma maneira não sistêmica, desfavorecendo uma visão integrada da sustentabilidade do negócio.

Outro ponto controverso é a adequação do modelo de reporte da GRI para organizações de todos os portes. Enquanto Calabrese et al. (2016) consideram as diretrizes da GRI para empresas de todas as dimensões e setores, Ferreira-Quilice e Caldana (2015) apontam que o modelo é mais apropriado para grandes empresas, até mesmo pelo alto custo para produção dos relatórios de sustentabilidade, o que é indicado como ponto negativo nesta revisão. 


\section{CONSIDERAÇÕES FINAIS}

Em consonância com o conceito de "desenvolvimento sustentável", muitas organizações vêm divulgando sua relação com a questão da sustentabilidade por meio de relatórios. As diretrizes da GRI para produção desses relatórios se destacam, sendo a mais utilizada e com maior credibilidade no mundo. Este estudo teve como objetivo identificar, por meio de revisão da literatura, pontos positivos e negativos dos relatórios GRI. Buscou-se contribuir preenchendo uma lacuna na literatura sobre estudos desse tipo. Embora haja muitos artigos que tratam sobre a GRI, carece-se de estudos que reúnam os pontos fortes e fracos desse modelo de reporte.

Para o alcance do objetivo do artigo foram selecionados 55 artigos em língua inglesa ou portuguesa. A revista "Journal of Cleaner Production" destacou-se pelo número de trabalhos dedicados ao tema. No entanto, o assunto se caracteriza como multidisciplinar, sendo publicado por revistas das mais variadas áreas.

Os pontos positivos foram divididos em quatro categorias: "Responsabilidade, transparência e legitimidade organizacional"; "Padronização e comparabilidade"; "Popularidade e credibilidade"; e "Abrangência". Já os pontos negativos em seis: "Incompletude"; "Padronização pouco rígida e dificuldade em estabelecer comparações", "Greenwashing"; "Diferentes expectativas dos stakeholders"; "Alto custo de desenvolvimento do relatório"; e "Falta de integração entre indicadores".

Observa-se que há certos pontos de controvérsias entre autores, a exemplo daqueles que indicam a "padronização e comparabilidade" como ponto positivo, enquanto outros apontam "padronização pouco rígida e dificuldade em estabelecer comparações" como ponto negativo. Uma sugestão seria a de enrijecer o padrão já existente, o que favoreceria 0 estabelecimento comparações.

Outra questão controversa é o fato de que alguns autores apontam como vantagem a abrangência dos indicadores GRI, enquanto outros relatam a falta de alguns deles. Outros autores ainda apontam a falta de integração entre os indicadores, o que acaba por produzir um relatório com uma abordagem reducionista do conceito de desenvolvimento sustentável.

Muitos autores argumentam que $\mathrm{o}$ uso do modelo favorece a responsabilidade, a transparência e a legitimidade organizacional junto às partes interessadas. Claro que não se pode deixar de salientar o problema do "greenwashing", pois há na literatura indícios de que empresas não retratam a realidade em seus relatórios, utilizando-os como ferramenta de marketing.

Apesar do rigor no método de seleção das referências, como limitação deste trabalho indica-se o fato de terem sido incluídos apenas artigos revisados por pares, publicados em periódicos, e em língua inglesa ou portuguesa. Além disso, apenas algumas bases de dados foram selecionadas e considerou-se apenas estudos de 2014 em diante. Todas essas questões limitam o número de artigos encontrados, podendo-se excluir contribuições importantes nessa área de conhecimento. Para compensar tais limitações, buscou-se cobrir as principais bases de dados, bem como selecionar um período razoável para obtenção de estudos mais recentes. 
Como sugestão para pesquisas futuras, recomenda-se um aprofundamento na questão da materialidade, uma vez que alguns dos autores citados nesta revisão indicaram ser um ponto crítico dos relatórios GRI e há poucos estudos sobre o tema. Poderia ser investigada a questão de como o princípio da materialidade pode ser desvirtuado para praticar o "greenwashing", ou seja, como manipular o desejo das partes interessadas para se produzir um instrumento meramente publicitário, fugindo totalmente da proposta de relatar o compromisso organizacional com a sustentabilidade. 


\title{
Positive and negative aspects of sustainability reports in the global reporting initiative model: national and international literature review
}

\begin{abstract}
Considering the increasing participation of companies around the world by the Global Reporting Initiative (GRI) reporting model to produce sustainability reports, the objective of this article is to identify the positive and negative aspects of this model. To do so, a review of the literature was carried out, searching for national and international articles in databases in the Portal de Periódicos da Coordenação de Aperfeiçoamento de Pessoal de Nivel Superior. We selected 55 articles that brought positive and / or negative points of the sustainability reports in the GRI reporting model. It was observed that the journal "Journal of Cleaner Production" stood out in relation to the number of publications on the subject, with 25 articles of the total. The highlights of the research were grouped in the following topics: "Responsibility, transparency and organizational legitimacy"; "Standardization and comparability"; "Popularity and credibility"; and "Scope". On the other hand, the main points were: "Incompleteness"; "Non-rigid standardization and difficulty in establishing comparisons", "Greenwashing"; "Different stakeholder expectations"; "High cost of developing the report"; and "Lack of integration between indicators". It was sought to contribute by filling a gap in the literature on such studies, which gathered the strengths and weaknesses of the GRI model, observing also certain points of controversy among authors. As a suggestion for future research, it is recommended a deepening in relation to the principle of materiality, considered critical by some authors present in this review and little discussed in the literature.
\end{abstract}

KEYWORDS: Sustainable development. Sustainability report. GRI. Corporate social responsibility. 
ADAMS, C. A. The International Integrated Reporting Council: A call to action. Critical Perspectives On Accounting, v. 27, p.23-28, 2015. crossref

ALCARAZ-QUILES, F. J.; NAVARRO-GALERA, A.; ORTIZ-RODRÍGUEZ, D. Transparency over sustainability in regional governments: the case of Spain. Convergencia, v. 24, n. 73, p.113-140, 2017.

ARAS, G.; TEZCAN, N.; FURTUNA, O. K. Multidimensional comprehensive corporate sustainability performance evaluation model: Evidence from an emerging market banking sector. Journal of Cleaner Production, v. 185, p.600609,2018 . crossref

ARENA, M.; AZZONE, G.; MAPELLI, F. What drives the evolution of Corporate Social Responsibility strategies? An institutional logics perspective. Journal of Cleaner Production, v. 171, p.345-355, 2018. crossref

BEBBINGTON, J. Sustainable Development: A Review of the International Development, Business and Accounting Literature. Ssrn Electronic Journal, p.128157, 2001. crossref

BJ $\varnothing \mathrm{RN}, \mathrm{A}$. et al. Is Earth recognized as a finite system in corporate responsibility reporting? Journal of Cleaner Production, v. 163, p.106-117, 2017. crossref

BLASS, A. P. et al. Measuring environmental performance in hospitals: A practical approach. Journal of Cleaner Production, v. 142, p.279-289, 2017. crossref

BONSÓN, E.; BEDNÁROVÁ, M. CSR reporting practices of Eurozone companies. Revista de Contabilidad, v. 18, n. 2, p.182-193, 2015. cross ref

BORGERT, T. et al. Initiating sustainability assessments: Insights from practice on a procedural perspective. Environmental Impact Assessment Review, v. 72, p.99107, 2018. crossref

BORGES, M. L. et al. Corporate Social Responsibility (CSR) practices developed by Brazilian companies: an exploratory study. International Journal of Sustainable Development e World Ecology, v. 25, n. 6, p.509-517, 2017. crossref

BOUTEN, L. et al. Corporate social responsibility reporting: A comprehensive picture?. Accounting Forum, v. 35, n. 3, p.187-204, 2011. crossref

BROOKS, C.; OIKONOMOU, I. The effects of environmental, social and governance disclosures and performance on firm value: A review of the literature in accounting and finance. The British Accounting Review, v. 50, n. 1, p.1-15, 2018. 
BRUSCA, I.; LABRADOR, M.; LARRAN, M. The challenge of sustainability and integrated reporting at universities: A case study. Journal of Cleaner Production, v. 188, p.347-354, 2018. crossref

BURGWAL, D. van de; VIEIRA, R. J. O. Determinantes da divulgação ambiental em companhias abertas holandesas. Revista Contabilidade e Finanças, v. 25, n. 64, p.60-78, 2014. crossref

BURRITT, R; SCHALTEGGER, S. Accounting towards sustainability in production and supply chains. The British Accounting Review, v. 46, n. 4, p.327-343, 2014. crossref

CALABRESE, A et al. A fuzzy analytic hierarchy process method to support materiality assessment in sustainability reporting. Journal of Cleaner Production, v. 121, p.248-264, 2016. crossref

CAMPOS, L. M. de S. et al. Relatório de sustentabilidade: perfil das organizações brasileiras e estrangeiras segundo o padrão da Global Reporting Initiative. Gestão e Produção, v. 20, n. 4, p.913-926, 2013. crossref

CORNELIUS, N.; WALLACE, J.; TASSABEHJI, R. An Analysis of Corporate Social Responsibility, Corporate Identity and Ethics Teaching in Business Schools. Journal of Business Ethics, v. 76, n. 1, p.117-135, 2007. crossref

DANOUCARAS, A. N.; WOODLEY, A. P.; MORAN, C. J. The robustness of mine water accounting over a range of operating contexts and commodities. Journal of Cleaner Production, v. 84, p.727-735, 2014. crossref

DE MARTINI JUNIOR, L.; SILVA, E. R. da; MATTOS, U. Análise da Transparência Corporativa através dos Relatórios de Sustentabilidade com base na Global Reporting Initiative de Empresas do Setor Brasileiro de Energia Elétrica. Sistemas e Gestão, v. 9, n. 1, p.34-46, 2014. crossref

DILLING, P. F. A.; HARRIS, P. Reporting on long-term value creation by Canadian companies: A longitudinal assessment. Journal of Cleaner Production, v. 191, p.350-360, 2018. crossref

DISSANAYAKE, D.; TILT, C.; XYDIAS-LOBO, M. Sustainability reporting by publicly listed companies in Sri Lanka. Journal of Cleaner Production, v. 129, p.169-182, 2016. crossref

DRAGOMIR, V. D. How do we measure corporate environmental performance? A critical review. Journal of Cleaner Production, v. 196, p.1124-1157, 2018.

crossref 
ELKINGTON, J. Cannibals with Forks: The Triple Bottom Line of the 21st Century Business. Oxford: Capstone, 1997. crossref

ETZION, D. et al. Unleashing sustainability transformations through robust action. Journal of Cleaner Production, v. 140, 167-178, 2017. crossref

FEDOROVA, E.; PONGRÁCZ, E. Cumulative social effect assessment framework to evaluate the accumulation of social sustainability benefits of regional bioenergy value chains. Renewable Energy, v. 131, p.1073-1088, 2019. crossref

FERNANDEZ-FEIJOO, B.; ROMERO, S.; RUIZ, S. Commitment to Corporate social responsibility measured through global reporting initiative reporting: factors affecting the behavior of companies. Journal of Cleaner Production, v. 81, p.244254, 2014. crossref

FERREIRA-QUILICE, T.; CALDANA, C. F. Aspectos negativos de reporte proposto pela GRI: a opinião das organizações que reportam. Revista de Administração, v. 50 , n. 4, p.405-415, 2015. crossref

FIFKA, M. S.; POBIZHAN, M. An institutional approach to corporate social responsibility in Russia. Journal of Cleaner Production, v. 82, p.192-201, 2014. crossref

FONSECA, A.; MCALLISTER, M. L.; FITZPATRICK, P. Sustainability reporting among mining corporations: a constructive critique of the GRI approach. Journal of Cleaner Production, v. 84, p.70-83, dez. 2014. crossref

FRITZ, M. M. C.; SCHÖGGL, J.; BAUMGARTNER, R. J. Selected sustainability aspects for supply chain data exchange: Towards a supply chain-wide sustainability assessment. Journal of Cleaner Production, v. 141, p.587-607, 2017. crossref

FUENTE, J. A.; GARCÍA-SÁNCHEZ, I. M.; LOZANO, M. B. The role of the board of directors in the adoption of GRI guidelines for the disclosure of CSR information. Journal of Cleaner Production, v. 141, p.737-750, 2017. crossref

FUISZ-KEHRBACH, S. A three-dimensional framework to explore corporate sustainability activities in the mining industry: Current status and challenges ahead. Resources Policy, v. 46, p.101-115, 2015. crossref

GIL, A. C. Métodos e técnicas da pesquisa social. 5. ed. São Paulo: Atlas, 2007.

GONZÁLEZ, M. et al. Modeling sustainability report scoring sequences using an attractor network. Neurocomputing, v. 168, p.1181-1187, 2015. crossref 
GRI - GLOBAL REPORTING INITATIVE. G4 Sustainability Reporting Guidelines: Reporting Principles and Standard Disclosures. Amsterdam: GRI, 2015.

GRI - GLOBAL REPORTING INITIATIVE. G4 to Standards: transition complete. 2018. Disponível em: <https://www.globalreporting.org/information/news-andpress-center/Pages/G4-to-Standards-transition-complete.aspx>. Acesso em: 16 ago. 2019.

GRI - GLOBAL REPORTING INITIATIVE. GRI's History. 2019a. Disponível em: $<$ https://www.globalreporting.org/information/about-gri/grihistory/Pages/GRI's\%20history.aspx>. Acesso em: 16 ago. 2019.

GRI - GLOBAL REPORTING INITIATIVE. Sustainability Disclosure Database. 2019b. Disponível em: <http://database.globalreporting.org>. Acesso em: 16 ago. 2019.

HSU, C.; LEE, W.; CHAO, W. Materiality analysis model in sustainability reporting: a case study at Lite-On Technology Corporation. Journal of Cleaner Production, v. 57, p.142-151, 2013. crossref

JACKSON, J.; BELKHIR, L. Assigning firm-level GHGE reductions based on national goals - Mathematical model e empirical evidence. Journal of Cleaner Production, v. 170, p.76-84, 2018. crossref

KOSKELA, M. Occupational health and safety in corporate social responsibility reports. Safety Science, v. 68, p.294-308, 2014. crossref

LACY, P. et al. A New Era of Sustainability: UN Global Compact, Acccenture CEO Study 2010. New York: United Nations, 2010.

LEONG, S. et al. Mine site-level water reporting in the Macquarie and Lachlan catchments: a study of voluntary and mandatory disclosures and their value for community decision-making. Journal of Cleaner Production, v. 84, p.94-106, 2014. crossref

LIMA, R. G. de et al. A sustainability evaluation framework for Science and Technology Institutes: an international comparative analysis. Journal of Cleaner Production, v. 125, p.145-158, 2016. crossref

LIUBACHYNA, A.; SECCO, L.; PETTENELLA, D. Reporting practices of State Forest Enterprises in Europe. Forest Policy And Economics, v. 78, p.162-172, 2017. crossref

LOCK, I.; SEELE, P. The credibility of CSR (corporate social responsibility) reports in Europe. Evidence from a quantitative content analysis in 11 countries. Journal of Cleaner Production, v. 122, p.186-200, 2016. crossref 
MACHADO, M. C. et al. Sustainability in Information Technology: an analysis of the aspects considered in the model Cobit. Journal of Information Systems and Technology Management, v. 14, n. 1, p.88-110, 2017. crossref

MANCINI, L.; SALA, S. Social impact assessment in the mining sector: Review and comparison of indicators frameworks. Resources Policy, v. 57, p.98-111, 2018. crossref

MARIMON, F. et al. The worldwide diffusion of the Global Reporting Initiative: what is the point? Journal of Cleaner Production, v. 33, p.132-144, 2012.

\section{crossref}

MASCENA, K.; FISCHMANN, A.; BOAVENTURA, J. Stakeholder Prioritization in Brazilian Companies Disclosing GRI Reports. Brazilian Business Review, v. 15, n. 1, p.17-32, 2018. crossref

MELÉ, D.; DEBELUUH, P.; ARRUDA, M. C. Corporate Ethical Policies in Large Corporations in Argentina, Brazil and Spain. Journal of Business Ethics, v. 63, n. 1, p.21-38, 2006. crossref

MICHELON, G.; PILONATO, S.; RICCERI, F. CSR reporting practices and the quality of disclosure: An empirical analysis. Critical Perspectives on Accounting, v. 33, p.59-78, 2015. crossref

MILNE, M. J.; GRAY, R. W(h)ither Ecology? The Triple Bottom Line, the Global Reporting Initiative, and Corporate Sustainability Reporting. Journal of Business Ethics, v. 118, n. 1, p.13-29, 2012. crossref

MORIOKA, S. N.; CARVALHO, M. M. de. Discutindo sustentabilidade no contexto de negócios e em relatórios de desempenho: análise de estudos de caso brasileiros. Gestão e Produção, v. 24, n. 3, p.514-525, 2017. crossref

MURILLO, D.; LOZANO, J. M. SMEs and CSR: An Approach to CSR in their Own Words. Journal of Business Ethics, v. 67, n. 3, p.227-240, 2006. crossref

NASCIMENTO, G.; ARAUJO, C. A. S.; ALVES, L. A. Corporate sustainability practices in accredited Brazilian hospitals: a degree-of-maturity assessment of the environmental dimension. Revista de Administração, v. 52, n. 1, p.26-35, 2017. crossref

OLIVEIRA, M. de A. S. et al. Relatórios de sustentabilidade segundo a Global Reporting Initiative (GRI): uma análise de correspondências entre os setores econômicos brasileiros. Production, v. 24, n. 2, p.392-404, 2013. crossref

ÖZDEMIR, E. D. et al. A confusion of tongues or the art of aggregating indicators-Reflections on four projective methodologies on sustainability 
measurement. Renewable and Sustainable Energy Reviews, v. 15, n. 5, p.23852396, 2011. crossref

PIMENTA, H. C. D.; MORAIS, A. C. S. de. Avaliação de práticas de Responsabilidade Social Corporativa em supermercados de Natal-RN. Revista Gestão Industrial, v. 10, n. 2, p.430-455, 2014. crossref

PORTER, Michael E.; KRAMER, Mark R. Strategy and society: the link between competitive advantage and corporate social responsibility. 2006. Disponível em: <https://edisciplinas.usp.br/pluginfile.php/3725941/mod_resource/content/1/3. \%20PORTER_strategy\%20\%20society\%202006.pdf>. Acesso em: 16 ago. 2019.

RAHDARI, A. H.; ROSTAMY, A. A. A. Designing a general set of sustainability indicators at the corporate level. Journal of Cleaner Production, v. 108, p.757771, 2015. cross ref

REBElATO, M. G.; ALMEIDA, A. Z. de; RODRIGUES, A. M. Desempenho Ambiental de Instituições Financeiras: um estudo comparativo entre os cinco principais bancos brasileiros. Revista Gestão Industrial, v. 11, n. 1, p.120-144, 2015. crossref

ROBERTS, S.; LAWSON, R.; NICHOLLS, J. Generating Regional-Scale Improvements in SME Corporate Responsibility Performance: Lessons from Responsibility Northwest. Journal of Business Ethics, v. 67, n. 3, p.275-286, 2006. crossref

ROBINSON, J. Squaring the circle? Some thoughts on the idea of sustainable development. Ecological Economics, v. 48, n. 4, p.369-384, 2004. crossref

RUIZ-LOZANO, M; TIRADO-VALENCIA, P. Do industrial companies respond to the guiding principles of the Integrated Reporting framework? A preliminary study on the first companies joined to the initiative. Revista de Contabilidad, v. 19, n. 2, p.252-260, 2016. crossref

SAMUDHRAM, A. et al. Towards a new paradigm: Activity level balanced sustainability reporting. Applied Ergonomics, v. 57, p.94-104, 2016. crossref

SCHMELTZ, L. Introducing value-based framing as a strategy for communicating CSR. Social Responsibility Journal, v. 10, n. 1, p.184-206, 2014. crossref

SCHOLL, C. A.; HOURNEAUX JUNIOR, F.; GALLELI, B. Sustentabilidade organizacional: aplicação de índice composto em uma empresa do setor químico. Gestão e Produção, v. 22, n. 4, p.695-710, 2015. crossref

SEARCY, C.; BUSLOVICH, R. Corporate Perspectives on the Development and Use of Sustainability Reports. Journal of Business Ethics, v. 121, n. 2, p.149-169, 2013. crossref 
SIEW, R. Y. J. A review of corporate sustainability reporting tools (SRTs). Journal of Environmental Management, v. 164, p.180-195, 2015. crossref

SILVESTRE, W. J.; AMARO, A. Sustentabilidade corporativa: avaliação híbrida do Triple Bottom Line. Revista de Gestão dos Países de Língua Portuguesa, v. 13, n. 4, p.19-29, 2014.

SMITH, R. L.; RUIZ-MERCADO, G. J.; GONZALEZ, M. A. Using GREENSCOPE indicators for sustainable computer-aided process evaluation and design. Computers e Chemical Engineering, v. 81, p.272-277, 2015. crossref

SOARES, D. A. S. da R. et al. Organizational culture and sustainability in Brazilian electricity companies. Rausp Management Journal, v. 53, n. 4, p.488-506, 2018. crossref

SOUZA, J. P. E.; ALVES, J. M. Lean-integrated management system: A model for sustainability improvement. Journal of Cleaner Production, v. 172, p.2667-2682, 2018. crossref

SPUERK, S.; DROBE, M.; LOTTERMOSER, B. G. Evaluating resource efficiency at major copper mines. Minerals Engineering, v. 107, p.27-33, 2017. crossref

TALBOT, D.; BOIRAL, O. GHG Reporting and Impression Management: An Assessment of Sustainability Reports from the Energy Sector. Journal of Business Ethics, v. 147, n. 2, p.367-383, 2018. crossref

TSALIS, T.; AVRAMIDOU, A.; NIKOLAOU, I. E. A social LCA framework to assess the corporate social profile of companies: Insights from a case study. Journal of Cleaner Production, v. 164, p.1665-1676, 2017. crossref

VELEVA, V.; BODKIN, G.; TODOROVA, S. The need for better measurement and employee engagement to advance a circular economy: Lessons from Biogen's "zero waste" journey. Journal of Cleaner Production, v. 154, p.517-529, 2017. crossref

VOGT, M. et al. Relationship between determinant factors of disclosure of information on environmental impacts of Brazilian companies. Estudios Gerenciales, v. 33, n. 142, p.24-38, 2017. crossref

WAGNER, R.; SEELE, P. Uncommitted Deliberation? Discussing Regulatory Gaps by Comparing GRI 3.1 to GRI 4.0 in a Political CSR Perspective. Journal of Business Ethics, v. 146, n. 2, p.333-351, 2017. crossref

WCED - WORLD COMMISSION ON ENVIRONMENT AND DEVELOPMENT. Our Common Future. Oslo: United Nations, 1987. 
WEBER, O.; SAUNDERS-HOGBERG, G. Water management and corporate social performance in the food and beverage industry. Journal of Cleaner Production, v. 195, p.963-977, 2018. crossref

ZHOU, Y.; LI, Y. P.; HUANG, G. H. Integrated modeling approach for sustainable municipal energy system planning and management - A case study of Shenzhen, China. Journal of Cleaner Production, v. 75, p.143-156, 2014. crossref

VIEIRA, I.L. et al. Pontos positivos e negativos dos relatórios de sustentabilidade no modelo global reporting initiative: revisão da literatura nacional e internacional. R. Gest. Industr., Ponta Grossa, v. 16, n. 2, p. 21-46, Abr./Jun. 2020. Disponível em: https://periodicos.utfpr.edu.br/revistagi

Correspondência:

Igor Laguna Vieira

Rua São Francisco Xavier, 524, Maracanã, Rio de Janeiro - RJ - Cep 20550-900 - Pavilhão Reitor João Lyra Filho - 50 andar - sala 5002 - bloco A - Telefone: (21) 2334-0512 ramal 20

Direito autoral: Este artigo está licenciado sob os termos da Licença Creative Commons-Atribuição 4.0 Internacional.

\section{(c) (1)}

